\title{
The Effects of Moderate Exercise on Ethanol-Induced Gastric Injuries in Rats
}

\author{
Hadis Rostami-Motamed, ${ }^{1}$ Majid Taati, ${ }^{2,}$ Omid Dezfoulian, ${ }^{3}$ Masoud Alirezaei, ${ }^{4}$ and \\ Mehrnoush Moghaddasi ${ }^{5}$
}

${ }^{1}$ Department of Physiology, Faculty of Sciences, Hamedan Branch, Islamic Azad University, Hamedan, IR Iran

${ }_{2}^{2}$ Department of Physiology, School of Veterinary Medicine, Lorestan University, Khorramabad, IR Iran

3 Department of Pathology, School of Veterinary Medicine, Lorestan University, Khorramabad, IR Iran

${ }^{4}$ Department of Pathology, School of Veterinary Medicine, Lorestan University, Khorramabad, IR Iran

5 Department of Physiology, Razi Herbal Medicines Research Center, Lorestan University of Medical Sciences, Khorramabad, IR Iran

*Corresponding author: Majid Taati, Department of Physiology, School of Veterinary Medicine, Lorestan University, Khorramabad, IR Iran. E-mail: taatimajid@yahoo.com

Received:April 26, 2014; Accepted: August 5, 2014

\begin{abstract}
Background: The role of oxygen-derived free radicals in the development of pathogenesis in acute gastric lesions induced by ethanol is well known. The beneficial effects of physical training on the antioxidant defenses of numerous tissues have been proved.

Objectives:The present study was designed to evaluate the effects of exercise training on ethanol-induced gastric mucosal injuries in rats. Materials and Methods: In this experimental study, 28 Sprague-Dawley male rats were divided into 4 equal groups: sedentary control, exercise control, sedentary plus ethanol and exercise plus ethanol groups. The rats were subjected to moderate treadmill exercise protocol for 30 consecutive days (one hour per day). Thereafter, ethanol-treated groups received absolute ethanol (1 mL/rat) by gavage to induce gastric ulcer. Antioxidant status and lipid peroxidation of stomach wall were measured by evaluation of glutathione peroxidase (GPx), catalase activities (CAT) and thiobarbituric acid reactive substances (TBARS) concentration, respectively.

Results: The histopathological findings of this study showed that moderate exercise training significantly inhibited the ethanol-induced gastric lesions in rats. Biochemical results also showed that administration of ethanol significantly decreased GPx activity and increased TBARS when compared to the sedentary control group $(\mathrm{P}<0.05)$. In contrast, the exercise plus ethanol group showed higher GPx activity and lower TBARS concentration when compared to the ethanol-treated rats $(\mathrm{P}<0.05)$. The catalase activity in ethanol-treated rats tended to be decreased when compared to control group.

Conclusions: It seems that the preventive effects of exercise training may be mediated through increasing GPx activity subsequently inhibition of lipid peroxidation in stomach wall of rats.
\end{abstract}

Keywords: Exercise; Ethanol; Stomach ulcer; Glutathione peroxidase

\section{Background}

There is extensive evidence documenting the health-promoting effects of exercise [1]. Several reports have studied the useful effects of exercise on the gastrointestinal tract such as protective role against colon cancer, diverticular disease, cholelithiasis and constipation [2,3]. Although the mechanisms underlying these effects are not understood fully, altered gastrointestinal blood flow and motor function, neuroendocrine changes and mechanical effects are probably involved [4].

Ulcerative lesions of gastrointestinal tract are one of the major side effects associated with alcohol consumption [5]. Studies have shown alterations in the antioxidant status following ulceration, suggesting that reactive oxygen species (ROS) such as superoxide anion, hydrogen peroxide, and hydroxyl radical play a crucial role in the development of ethanol-induced ulceration in rats [6, 7]. ROS induce oxidative damage in membrane lipids and proteins and deplete glutathione level in gastric ulcers [6]. Indeed, "on one hand, ethanol administration reduces mucus production, gastric mucosal blood flow, bicarbonate secretion, endogenous glutathione and prostaglandin levels, and on the other hand it increases the release of histamine, the influx of calcium ions, the generation of free radicals and the production of leukotrienes" [8]. Several drugs are widely used to prevent or treat gastro-duodenal ulcers; these include $\mathrm{H}_{2}$-receptor antagonists (cimetidine), proton pump inhibitors (omeprazole, lansoprazole) and cytoprotectives (misoprostol) [9,10]. Antacids, e.g. aluminium hydroxide and magnesium hydroxide, are used often to neutralize excess gastric acidity in the stomach.

It is recognized that exercise affects oxidative metabolism. For example, Ziegler et al. [11] have shown that aerobic physical activity increases antioxidant enzyme activities, elevates resistance to oxidation of low density lipoproteins (LDL), and decreases levels of oxidized LDL in young and elderly patients with various forms of vascular diseases. In addition, plasma antioxidant levels are correlated with physical performance [12]. Exercise is also known to decrease levels of inflammatory mediators [13]. While regular exercise training is associated with numerous health benefits, it can be viewed as an intense physical stressor

Copyright (C) 2015, Zahedan University of Medical Sciences. This is an open-access article distributed under the terms of the Creative Commons Attribution-NonCommercial 4.0 International License (http://creativecommons.org/licenses/by-nc/4.0/) which permits copy and redistribute the material just in noncommercial usages, provided the original work is properly cited. 
leading to increased oxidative cellular damage, likely due to enhanced production of ROS [14]. It has been found that training prevents the appearance of some signs of exerciseinduced free radical generation [15]. While the effects of physical training on the antioxidant defenses of tissues such as liver, heart, brain, muscle and kidney have been investigated, the potential role of exercise in the gastrointestinal mucosal defense system is less well elucidated [16-19].

\section{Objectives}

The present study was designed to evaluate the effects of exercise training on ethanol-induced gastric mucosal injuries in rats. The effect of preconditioning exercise on ethanol-induced antioxidant enzymes activities and lipid peroxidation level alterations in gastric mucosa was investigated. In addition, the histological effects of the exercise were studied.

\section{Materials and Methods}

\subsection{Chemicals and Kits}

In this experimental study, ethanol (99.5\%) and thiobarbituric acid (TBA) were prepared from Merck Chemical Company (KGaA, Darmstadt, Germany). GPx kit was obtained via Randox ${ }^{\circledR}$ Company (Antrim, UK).

\subsection{Animals}

A total of 28 adult male Sprague-Dawley rats (weighing 220 $\pm 10 \mathrm{~g}$ ), were obtained from breeding colony of Lorestan University of Medical Sciences, Khorramabad, Iran. All rats were housed in a 12-hour light/dark cycle at $22-24^{\circ} \mathrm{C}$, with food and water ad libitum. All rats were treated humanely and in compliance with the recommendations of Animal Care Committee for the Lorestan University of Medical Sciences.

\subsection{Experimental Design and Treadmill Training}

The rats (7 rats in each group) were randomly selected in 4 equal groups:(A) sedentary control, (B) exercise control, (C) sedentary plus ethanol treatment and (D) exercise plus ethanol. While, groups B and D were subjected to treadmill exercise training for 4 consecutive weeks, groups A and C were not exercised. A modified human treadmill was applied. A shock grid was made into each channel to enforce the animals to run. The rats were subjected to treadmill exercise protocol for a total of 4 weeks: 30 minutes daily for 1 week thereafter 60 minutes of exercise for 3 additional weeks at a speed of $15 \mathrm{~m} /$ minute [19]. The rats were given a rest period of 5 minutes after 15 minutes of exercise the first week and then after 30 minutes in the last 3 weeks. All groups of rats had free access to standard rodent chow and water. At the end of exercise period, the rats were fasted and allowed access to water ad libitum for 24 hours before ethanol ingestion. Animals in groups A and B received orally (1 mL/rat) normal saline while groups $C$ and $D$ received absolute ethanol ( $1 \mathrm{~mL} / \mathrm{rat})$ by gavage to induce gastric ul- ceration [20]. One hour after saline and ethanol administration, all of animals were sacrificed upon light ether anesthesia (Dagenham, UK) by decapitation, and the stomachs were removed, inflated by injecting $2 \mathrm{~mL}$ of normal saline and opened along the greater curvature. Stomachs were gently rinsed with distilled water to remove gastric content and blood clots. The gastric tissues were removed for biochemical analysis from the antral portion of the stomachs and stored at $-70^{\circ} \mathrm{C}$ up to 2 months for determination of antioxidant status and lipid peroxidation.

\subsection{Histological Examination}

Immediately after macroscopic evaluation the stomachs were fixed in neutral buffered formalin (10\%) then routine histologic processing was carried out; $5-6 \mu \mathrm{m}$ sections were stained by Hematoxylin and Eosin method and were evaluated microscopically (Olympus $\mathrm{CH} 30$ ).

\subsection{Tissue Preparation for Biochemical Analysis}

The samples were thawed and manually homogenized in cold phosphate buffer $(0.1 \mathrm{M}, \mathrm{pH}=7.4$, containing $5 \mathrm{mM}$ EDTA) on liquid nitrogen, and debris were removed by centrifugation at $400 \times \mathrm{g}$ for 5 minutes (Centrifuge $5415 \mathrm{R}$; Rotofix 32A, Germany). Supernatants were recovered and used for protein measurement, antioxidant enzyme activities (GPx, CAT), and thiobarbituric acid-reactive substances (TBARS) concentrations. Protein content of tissue homogenates was determined using a colorimetric method of Lowry with bovine serum albumin as a standard [21].

\subsection{Measurement of GPx Activity}

The activity of GPx was evaluated with Randox ${ }^{\circledR}$ GPx detection kit according to the manufacturer's instructions. GPx catalyzes the oxidation of GSH by cumene hydroperoxide. In the presence of glutathione reductase and NADPH, the oxidized glutathione (GSSG) is immediately converted to the reduced form with a concomitant oxidation of NADPH to NADP+. The decrease in absorbance was measured spectrophotometrically (S2000 UV model; WPA, Cambridge, UK) against blank at $340 \mathrm{~nm}$. One unit (U) of GPx was defined as $1 \mu \mathrm{mol}$ of oxidized NADPH per minute per milligram of tissue protein. The GPX activity was expressed as U/mg of tissue protein.

\subsection{Measurement of Catalase Activity (CAT) Activity}

Tissue catalase activity was assayed according to the method we applied in our previous study [22]. The reaction mixture $(1 \mathrm{~mL})$ consisted of $50 \mathrm{mM}$ potassium phosphate $(\mathrm{pH}=$ 7.0), $19 \mathrm{mM} \mathrm{H}_{2} \mathrm{O}_{2}$ and a 20 - $50 \mu \mathrm{L}$ sample. The reaction was initiated by the addition of $\mathrm{H}_{2} \mathrm{O}_{2}$, and absorbance changes were measured at $240 \mathrm{~nm}\left(25^{\circ} \mathrm{C}\right)$ for 30 seconds. The molar extinction coefficient for $\mathrm{H}_{2} \mathrm{O}_{2}$ is $43.6 / \mathrm{M} . \mathrm{cm}$. The CAT activity was expressed in $\mu \mathrm{mol} \mathrm{H}_{2} \mathrm{O}_{2} /$ minute/mg of protein.

\subsection{Measurement of Lipid Peroxidation}

The amount of lipid peroxidation was indicated by the 
content of TBARS in the stomach. Tissue TBARS determined by following the production of thiobarbituric acid-reactive substances as described previously by Subbarao et al. [23]. In short, $40 \mu \mathrm{L}$ of homogenate was added to $40 \mu \mathrm{L}$ of $0.9 \% \mathrm{NaCl}$ and $40 \mu \mathrm{L}$ of deionized $\mathrm{H}_{2} \mathrm{O}$, resulting in a total reaction volume of $120 \mu \mathrm{L}$. The reaction was incubated at $37^{\circ} \mathrm{C}$ for 20 minutes and was stopped by the addition of $600 \mu \mathrm{L}$ of cold $0.8 \mathrm{M}$ hydrochloride acid, containing $12.5 \%$ trichloroacetic acid. Following the addition of $780 \mu \mathrm{L}$ of $1 \% \mathrm{TBA}$, the reaction was boiled for 20 minutes and then cooled at $4{ }^{\circ} \mathrm{C}$ for 1 hour. In order to measure the amount of TBARS produced by the homogenate, the cooled reaction was spun at $1,500 \times \mathrm{g}$ in a microcentrifuge for 20 minutes, and the absorbance of the supernatant was spectrophotometrically read at $532 \mathrm{~nm}$, using an extinction coefficient of $1.56 \times 105$ M.cm. The blanks for all of the TBARS assays contained an additional $40 \mu$ : of $0.9 \%$ $\mathrm{NaCl}$ instead of homogenate as just described. TBARS results were expressed as nmol/mg of tissue protein.

\subsection{Statistical Analysis}

Statistical analysis was performed using the statistical package SPSS-19. All variables were tested for normal and homogeneous variances by Leven's statistic test. The data were expressed as Mean \pm SEM and tested for significance by one-way analysis of variance (ANOVA) with Dunnett ttest as post hoc test. A calculated P value of less than 0.05 was considered statistically significant.

\section{Results}

Histological evaluation of gastric mucosa was shown in Figure 1. Administration of ethanol revealed highly expanded mucosal erosions in all animals (Figure $1 \mathrm{C}$ ). On microscopic examination, ethanol-treated rats showed mucosal necrosis of gastric epithelium and edema. While, rats that were trained with moderate exercise had comparatively better protection of the gastric mucosa against ethanol as seen by reduction in severity of gastric erosions and reduced or absent submucosal edema (Figure 1D).

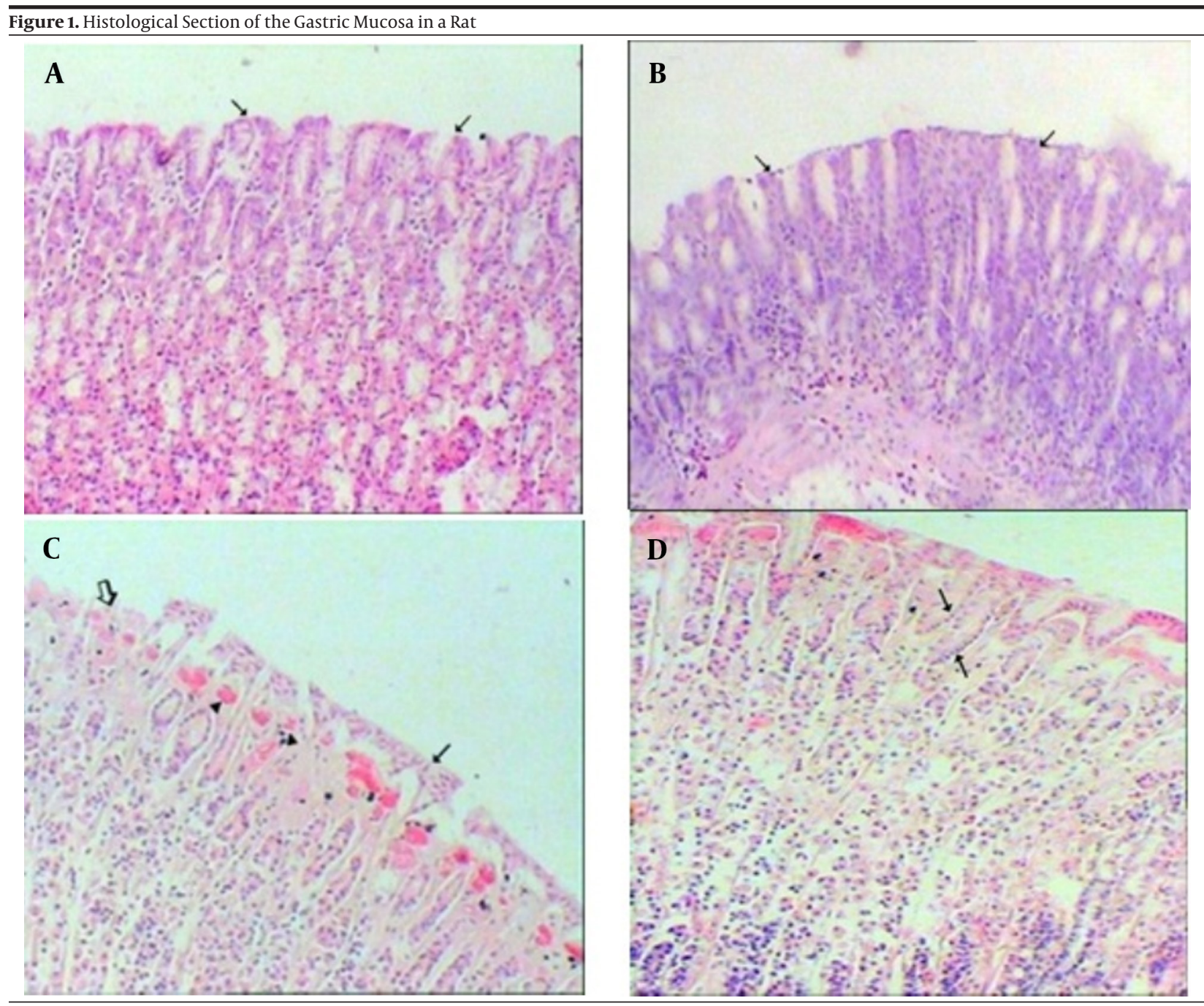

A: control group: The epithelial layer is intact (Arrows). B: exercise group: The mucosal layer is normal (Arrows). C: ethanol group: necrotizing epithelial cells (open arrow) in contrast to relatively normal cells (Arrow). Also the lamina propria is highly congested (Arrow heads). D: exercise plus ethanol group in which the epithelium has no change (Arrows), however in deep lamina propria the mononuclear cells are aggregated. (A - D) H and E, 40×. 
As shown in Figure 2, ethanol significantly reduced the GPx activity in gastric mucosa of ethanol-treated rats as compared to sedentary control group. There were significant differences between the ethanoltreated rats and exercise group. Exercise training increased GPx activity in exercise plus ethanol group significantly (Figure 2).

Although catalase (CAT) activity in gastric mucosa of ethanol treated rats was decreased, this reduction was not significant when compared to control group (Figure 3).

Figure 2. Comparison of Glutathione Peroxidase (GPx) Activity Among the Control and Treated Groups

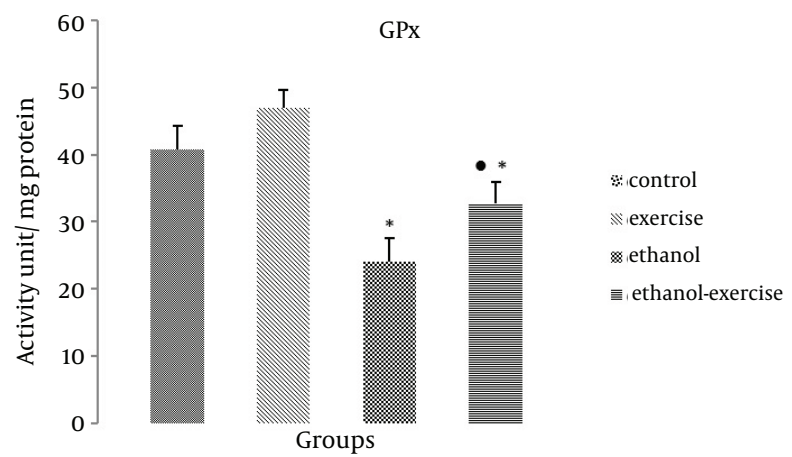

* Represents the significant difference between control with ethanol and ethanol plus exercise groups. - Represents the significant difference between ethanol and ethanol plus exercise groups. Values represent Mean \pm SEM of enzyme activity (U/mg protein of gastric tissue). $\mathrm{P}<0.05, \bullet \mathrm{P}<0.05$.

Figure 3. Comparison of Catalase (CAT) Activity Among the Groups

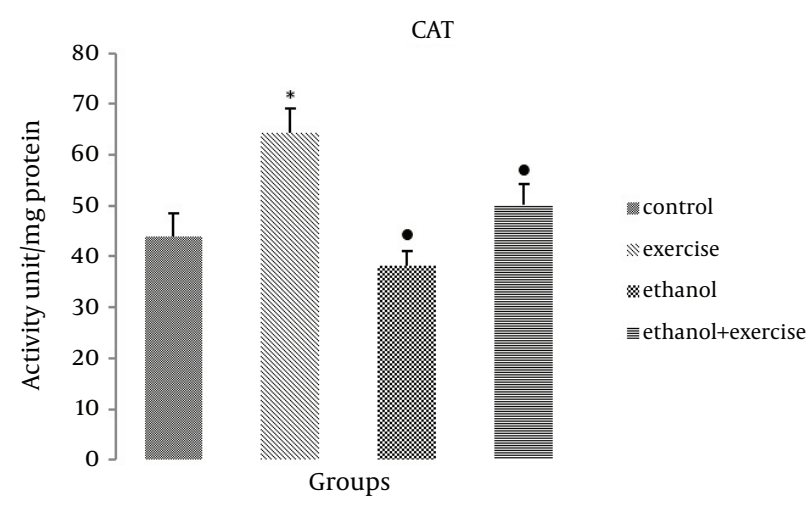

\footnotetext{
* Represents the significant difference between exercise and contro groups. - Represents the significant difference between exercise with ethanol and ethanol plus exercise groups. Values represent Mean \pm SEM of enzyme activity (unit/mg protein of gastric tissue). ${ }^{*} \mathrm{P}<0.05, \bullet \mathrm{P}<0.05$.
}

Exercise training increased the CAT activity in gastric mucosa significantly when compared to ethanol treated rats. Exercise training was not found to increase significantly CAT activity in exercise plus ethanol group (Figure 3).

Regarding lipid peroxidation, ethanol treatment significantly increased the TBARS level, and the exercise training resulted in a significant decrease of TBARS level in exercise plus ethanol group as compared to ethanol-treated group (Figure 4).

Figure 4. Comparison of Thiobarbituric Acid-Reactive Substances (TBARS) Concentration Among the Groups

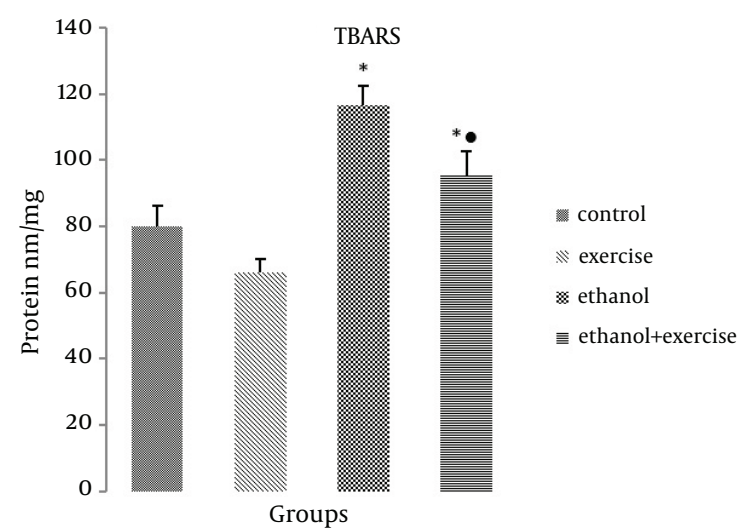

Represents the significant difference between control with ethanol and ethanol plus exercise groups. • represents the significant difference between ethanol and ethanol plus exercise groups. Values represent Mean \pm SEM of TBARS (nanomoles per milligram protein of gastric tissue). ${ }^{*} \mathrm{P}<0.05, \bullet \mathrm{P}<0.05$.

\section{Discussion}

In the present study activities of GPx and lipid peroxidation level of gastric mucosa was shown to increase and decrease respectively with the exercise. The present study also showed that the exercise reduced the ethanol induced gastric erosion and submucosal edema. It is wellknown that ethanol acts as a damaging agent to gastric mucosa and has been widely used for the experimental evaluation of anti-ulcer activities of different chemicals $[5,8,20]$. It has been suggested that ethanol induced gastric mucosal lesions may be attributed to the mechanisms such as: (1) increased oxygen-derived free radicals, (2) decreased concentration of reduced glutathione contents in gastric mucosa, (3) direct damage to the mucin layer or mucin synthesis and (4) gastric cell's apoptosis $[8,24]$. Gastric mucosal integrity is maintained by a dynamic process of cell death and cell proliferation. Among various factors involved in gastric mucosal lesions, oxidative damage and apoptotic cell death play significant roles in the loss of gastric mucosal integrity caused by 
various ulcerogens $[6,25]$. The generation of ROS plays a major role in the development of multiple pathologies, such as gastritis, peptic ulcerations or gastric adenocarcinoma $[24,26]$. Indeed, ulcers develop when oxidative damage and apoptosis predominate over the healing process by cell proliferation; in contrast, various growth factors, nitric oxide, endothelin, mitogen-activated protein kinases, and some of the oncogene such as c-myc, c-Ha-ras, and c-fos participate in ulcer improvement [7, 8]. To prevent the free radical propagation effect, the body uses antioxidants to stop the biochemical chain reaction. Antioxidants are compounds that dispose of reactive oxygen species by scavenging them, suppressing their formation, or opposing their actions [12]. Antioxidant mechanisms are complex and multifactorial. Antioxidants include the enzymes like GPx, superoxide dismutase (SOD), and CAT. These enzymes initiate sequences of reactions that can quickly convert oxygen radicals into harmless water. Other categories of antioxidants include the water-soluble and lipid soluble antioxidants, such as ascorbic acid and vitamin E, respectively. GPx and CAT can decompose $\mathrm{H}_{2} \mathrm{O}_{2}$ to water. Although $\mathrm{H}_{2} \mathrm{O}_{2}$ is not a particularly reactive product, it may be reduced to the highly reactive metabolites hydroxyl radicals and/or single oxygen [20,27].

Our results showed that administration of ethanol decreased CAT and GPX activities and enhanced TBARS concentration in gastric mucosa. These data are in agreement with previous studies that have suggested ethanol intoxication generally impairs the gastric antioxidant defense system and induces lipid peroxidation in experimental animals [5, 7]. The present findings for the first time showed that the moderate exercise training attenuates lesions in rat stomach caused by the application of noxious agents such as ethanol and that this protective effect is accompanied by the increase in CAT and GPx activities and reduction of TBARS concentration, as an index for lipid peroxidation process. It is widely accepted that lipid peroxidation is a mechanism of cellular injury. In this process polyunsaturated fatty acids in the cell membrane of living organisms are attacked by free radicals in the presence of molecular oxygen, a chain of chemical reactions can occur, eventually leading to the disintegration of fatty acid and formation of hydrocarbon gases (e.g. pentane) and aldehydes (e.g. malondialdehyde) [28]. In a previous study we demonstrated that substances with antioxidant properties may protect gastric mucosa against ethanol damaging effects [20]. Therefore, these results are in agreement with the previous report.

It is interesting to note that exhaustive exercise has been reported to increase reactive oxygen species, leading to oxidative stress [29]. Therefore, in this study animals were trained with a moderate exercise program [19]. There are numerous mechanisms that have been linked to the production of free radicals in physical exercise. The first involves hyperoxic injury that may oc- cur in highly intense aerobic exercise. Enhanced oxygen consumption may result in a flux of oxygen into the mitochondria that may lead to the formation of superoxide radicals and other more harmful radicals [30]. Another source of free radical production during aerobic exercise is the situation known as ischemia reperfusion injury. During exercise many organs, such as the liver, kidneys, and the splanchnic region, may experience hypoxia. This hypoxia is due to the shunting of blood to working muscles [29]. Ischemia results in a decrease in oxygen and substrate availability. The lack of adenosine triphosphate (ATP) due to the inability of anaerobic means to maintain pace with energy demands results in damaging effects [15]. The breakdown of ATP and the activation of xanthine oxidase from xanthine dehydrogenase during ischemia have been related to production of free radicals and tissue damage during reperfusion. In reperfusion, xanthine oxidase catalysed xanthine to uric acid and toxic reactive oxygen metabolites [27]. Because the regular exercise does not induce functional deterioration and, suitably graded, causes benefits such as adjunctive therapy, even in the treatment of patients with chronic heart failure, an exercise adaptation counteracting the effects of physical stress must take place. This apparently paradoxical outcome of different exercise protocols has been proposed to be related to upregulation of the antioxidative and repair capacities of cells that are induced by mild oxidative stress [13]. It has been, furthermore, found that training prevents the appearance of some signs of exercise-induced free radical generation [15]. Exercise at moderate intensity, when repeated, induces several changes at the cellular level that may counteract the increased ROS generation more efficiently [19]. Studies with animal models suggest that the intermediary metabolism adapts to the increasing demand of energy generation with a higher concentration of relevant enzymes in mitochondria by increasing the number of mitochondria rather than by increasing the enzymatic content in each mitochondrion [31]. As a result, each mitochondrion generates less prooxidant. Enzymatic antioxidative systems are also enhanced as a result of regular exercise [13].

Several animal studies suggest that moderate exercise increases antioxidant enzyme activity and attenuates oxidative stress in the liver, heart, brain, skeletal muscle, kidney and erythrocytes [17-19, 30, 31]. In this regard our finding showed that exercise significantly increased CAT activity in gastric tissue of animals which untreated with ethanol. It was indicated that regular physical exercise probably, due to the tachycardia-induced adaptation, has preconditioning effects to oxidative stress [32]. It is well known that rigorous physical exercise results in increased formation of reactive oxygen species (ROS) and these radicals might be stimulators of antioxidant enzymes in tissues [33]. Radak et al. [34] showed that administration of low levels of $\mathrm{H}_{2} \mathrm{O}_{2}$ results in increased resistance to oxidative stress. Low 
level treatment with $\mathrm{H}_{2} \mathrm{O}_{2}$ also increased protection against subsequent exposure to higher levels of $\mathrm{H}_{2} \mathrm{O}_{2}$ [34]. In fact, a significant correlation between antioxidant enzymes (SOD, CAT) and whole body maximal oxygen uptake (i.e. the best index for one's aerobic fitness level) has been reported. Moreover, resistance against oxidative stress preserves the normal $\mathrm{pH}$ level in tissues, thus preventing the production of loosely bound iron. This in turn protects tissue against damage due to ROS [35]. Therefore the stimulating effect of physical exercise on ROS generation is an important phenomenon of the exercise-induced adaptation process since it increases the resistance against prolonged oxidative stress and is a valuable physiological means of preconditioning the myocardium [33].

In conclusion, probably for the first time, this study showed that moderate exercise is able to prevent ethanolinduced gastric injuries via elevation of antioxidant status, and inhibition of lipid peroxidation in stomach wall.

\section{Acknowledgements}

This study is the result of university research proposal NO.17130509902016. The authors are thankful to Dr. B. Rasoulian for his kind cooperation in Razi Herbal Medicines Research Center, Lorestan University of Medical Science, Khorram Abad, Iran.

\section{Funding/Support}

Lorestan University, Khorramabad.

\section{References}

1. Garber CE, Blissmer B, Deschenes MR, Franklin BA, Lamonte MJ, Lee IM, et al. American College of Sports Medicine position stand Quantity and quality of exercise for developing and maintaining cardiorespiratory, musculoskeletal, and neuromotor fitness in apparently healthy adults: guidance for prescribing exercise. Med Sci Sports Exerc. 2011;43(7):1334-59.

2. de Oliveira EP, Burini RC. The impact of physical exercise on the gastrointestinal tract. Curr Opin Clin Nutr Metab Care. 2009;12(5):533-8.

3. Canelles P, Diago M, Tome A, Medina E, Orti E, Martinez A. [Physical exercise and gastric acid secretion]. Rev Esp Enferm Dig. 1990;77(3):179-84.

4. Simren M. Physical activity and the gastrointestinal tract. Eur J Gastroenterol Hepatol. 2002;14(10):1053-6.

5. Dekanski D, Ristić S, Mitrovic DM. Antioxidant effect of dry olive (Olea europaea L.) leaf extract on ethanol-induced gastric lesions in rats. Med J Nutr Metab. 2009;2(3):205-11.

6. Biswas K, Bandyopadhyay U, Chattopadhyay I, Varadaraj A, Ali E, Banerjee RK. A novel antioxidant and antiapoptotic role of omeprazole to block gastric ulcer through scavenging of hydroxyl radical. J Biol Chem. 2003;278(13):10993-1001.

7. de Barros MP, Sousa JP, Bastos JK, de Andrade SF. Effect of Brazilian green propolis on experimental gastric ulcers in rats. J Ethnopharmacol.2007;110(3):567-71.

8. Slomiany A, Morita M, Sano S, Piotrowski J, Skrodzka D, Slomiany BL. Effect of ethanol on gastric mucus glycoprotein synthesis, translocation, transport, glycosylation, and secretion. Alcohol Clin Exp Res. 1997;21(3):417-23.

9. Raskin JB, White RH, Jackson JE, Weaver AL, Tindall EA, Lies RB, et al. Misoprostol dosage in the prevention of nonsteroidal antiinflammatory drug-induced gastric and duodenal ulcers: a com- parison of three regimens. Ann Intern Med.1995;123(5):344-50.

10. Oliveira CP, Kassab P, Lopasso FP, Souza HP, Janiszewski M, Laurindo FR, et al. Protective effect of ascorbic acid in experimental gastric cancer: reduction of oxidative stress. World J Gastroenterol. 2003;9(3):446-8.

11. Ziegler S, Schaller G, Mittermayer F, Pleiner J, Mihaly J, Niessner A, et al. Exercise training improves low-density lipoprotein oxidability in untrained subjects with coronary artery disease. Arch Phys Med Rehabil. 2006;87(2):265-9.

12. Cesari M, Pahor M, Bartali B, Cherubini A, Penninx BW, Williams GR, et al. Antioxidants and physical performance in elderly persons: the Invecchiare in Chianti (InCHIANTI) study. Am J Clin Nutr. 2004;79(2):289-94.

13. Gleeson M, Bishop NC, Stensel DJ, Lindley MR, Mastana SS, Nimmo MA. The anti-inflammatory effects of exercise: mechanisms and implications for the prevention and treatment of disease. Nat Rev Immunol. 2011;11(9):607-15.

14. Moller P, Wallin H, Knudsen LE. Oxidative stress associated with exercise, psychological stress and life-style factors. Chem Biol Interact.1996;102(1):17-36.

15. Venditti P, Di Meo S. Antioxidants, tissue damage, and endurance in trained and untrained young male rats. Arch Biochem Biophys. 1996;331(1):63-8.

16. Chennaiah K, Basha KK, Sivasankar R, Ramaiah K, Reddy KS. Exercise prevents ethanol-induced oxidative stress: regulation of selected dehydrogenase activities in the skeletal muscle fibers of male albino rat with reference to aging. Asian J Anim Sci. 2011;6(1):8-13.

17. Toborek M, Seelbach MJ, Rashid CS, Andras IE, Chen L, Park M, et al. Voluntary exercise protects against methamphetamine-induced oxidative stress in brain microvasculature and disruption of the blood-brain barrier. Mol Neurodegener. 2013;8:22.

18. Asghar M, George L, Lokhandwala MF. Exercise decreases oxidative stress and inflammation and restores renal dopamine D1 receptor function in old rats. Am J Physiol Renal Physiol. 2007;293(3):F914-9.

19. Salim S, Sarraj N, Taneja M, Saha K, Tejada-Simon MV, Chugh G. Moderate treadmill exercise prevents oxidative stress-induced anxiety-like behavior in rats. Behav Brain Res. 2010;208(2):545-52.

20. Alirezaei M, Dezfoulian O, Neamati S, Rashidipour M, Tanideh N, Kheradmand A. Oleuropein prevents ethanol-induced gastric ulcers via elevation of antioxidant enzyme activities in rats. $J$ Physiol Biochem. 2012;68(4):583-92.

21. Lowry OH, Rosebrough NJ, Farr AL, Randall RJ. Protein measurement with the Folin phenol reagent. J Biol Chem.1951;193(1):265-75.

22. Taati M, Moghadasi M, Dezfoulian O, Asadian P, Kheradmand A Abbasi M, et al. The effect of ghrelin pretreatment on epididymal sperm quality and tissue antioxidant enzyme activities after testicular ischemia/reperfusion in rats. J Physiol Biochem. 2012;68(1):91-7.

23. Subbarao KV, Richardson JS, Ang LC. Autopsy samples of Alzheimer's cortex show increased peroxidation in vitro. J Neurochem. 1990;55(1):342-5.

24. Mutoh H, Hiraishi H, Ota S, Ivey KJ, Terano A, Sugimoto T. Role of oxygen radicals in ethanol-induced damage to cultured gastric mucosal cells. Am J Physiol Gastrointest Liver Physiol.1990;258(4):G603-9.

25. Das D, Banerjee RK. Effect of stress on the antioxidant enzymes and gastric ulceration. Mol Cell Biochem. 1993;125(2):115-25.

26. Odabasoglu F, Cakir A, Suleyman H, Aslan A, Bayir Y, Halici M et al. Gastroprotective and antioxidant effects of usnic acid on indomethacin-induced gastric ulcer in rats. J Ethnopharmacol. 2006;103(1):59-65.

27. Cuzzocrea S, Riley DP, Caputi AP, Salvemini D. Antioxidant therapy: a new pharmacological approach in shock, inflammation, and ischemia/reperfusion injury. Pharmacol Rev. 2001;53(1):135-59.

28. Li H, Rajendran GK, Liu N, Ware C, Rubin BP, Gu Y. SirT1 modulates the estrogen-insulin-like growth factor-1 signaling for postnatal development of mammary gland in mice. Breast Cancer Res. 2007;9(1):R1.

29. Rosa EF, Takahashi S, Aboulafia J, Nouailhetas VL, Oliveira MG. Oxidative stress induced by intense and exhaustive exercise impairs murine cognitive function. J Neurophysiol. 2007;98(3):1820-6.

30. Aksu I, Topcu A, Camsari UM, Acikgoz O. Effect of acute and chronic 


\section{Rostami-Motamed H et al.}

exercise on oxidant-antioxidant equilibrium in rat hippocampus, prefrontal cortex and striatum. Neurosci Lett. 2009;452(3):281-5.

31. Navarro A, Gomez C, Lopez-Cepero JM, Boveris A. Beneficial effects of moderate exercise on mice aging: survival, behavior, oxidative stress, and mitochondrial electron transfer. Am J Physiol Regul Integr Comp Physiol. 2004;286(3):R505-11.

32. Gielen S, Schuler G, Adams V. Cardiovascular effects of exercise training: molecular mechanisms. Circulation. 2010;122(12):1221-38.
33. McBride JM, Kraemer WJ. Free radicals, exercise, and antioxidants. J Strength Cond Res. 1999;13(2):175

34. Radak Z, Sasvari M, Nyakas C, Pucsok J, Nakamoto H, Goto S. Exercise preconditioning against hydrogen peroxide-induced oxidative damage in proteins of rat myocardium. Arch Biochem Biophys. 2000;376(2):248-51.

35. Leeuwenburgh C, Heinecke JW. Oxidative stress and antioxidants in exercise. Curr Med Chem. 2001;8(7):829-38. 